\title{
IL-1ß AND BMPS - INTERACTIVE PLAYERS OF CARTILAGE MATRIX DEGRADATION AND REGENERATION
}

\author{
Thomas Aigner, Stephan Soeder, Jochen Haag \\ Institute of Pathology, University of Leipzig, 04103 Leipzig, Germany
}

\begin{abstract}
Intact human adult articular cartilage is central for the functioning of the articulating joints. This largely depends on the integrity of its extracellular matrix, given the high loading forces during movements in particular in the weight-bearing joints. Unlike the first impression of a more or less static tissue, articular cartilage shows - albeit in the adult organism - a slow tissue turnover. Thus, one of the most important questions in osteoarthritis research is to understand the balance of catabolic and anabolic factors in articular cartilage as this is the key to understand the biology of cartilage maintenance and degeneration.

Anabolic and catabolic pathways are very much intermingled in articular cartilage. The balance between anabolism and catabolism is titrated on numerous levels, starting from the mediator-synthesizing cells which express either catabolic or anabolic factors. Also, on the level of the effector cells (i.e. chondrocytes) anabolic and catabolic gene expression compete for a balance of matrix homeostasis, namely the synthesis of matrix components and the expression and activation of matrix-degrading proteases. Also, there are multiple layers of intracellular cross-talks in between the anabolic and catabolic signalling pathways. Maybe the most important lesson from this overview is the notion that the anabolic-catabolic balance as such counts and not so much sufficient net anabolism or limited catabolism alone. Thus, it might be neither the aim of osteoarthritis therapy to foster anabolism nor to knock down catabolism, but the balance of anabolic-catabolic activities as a total needs proper titration and balancing.
\end{abstract}

Keywords: cartilage, matrix, cytokines, growth factors, osteoarthritis, anabolism, catabolism

Address for correspondence:

Thomas Aigner MD, DSc

Liebigstr. 26

D-04103 Leipzig, Germany

Tel.: +49-341-97 15036

Fax: +49-341-97 15019

E-mail: Thomas.Aigner@medizin.uni-leipzig.de

\section{Anabolism and catabolism: A balance of articular cartilage in the healthy - An imbalance in osteoarthritic cartilage degeneration}

Human adult articular cartilage is found as a whitish layer covering the interosseus surfaces of joints. It is an avascular and anerval tissue composed of a highly organized and specialized acellular component, the extracellular matrix, and a sparse cellular component, the chondrocytes (for review see Aigner and McKenna, 2002). Whereas the matrix is the functional component of articular cartilage, the chondrocytes are responsible for the homeostasis of this tissue.

The extracellular matrix of articular cartilage is composed mainly of two components defining its mechano-physical properties: the collagenous network (mainly collagen types II, IX, and XI, but also other noncollagenous molecules) is responsible for the tensile strength of the matrix and, therefore, of the cartilage; the proteoglycans (mainly aggrecan) are responsible for the osmotic swelling capacity and, thus, define the elastic properties of the cartilage tissue. Together, both components are able to dissipate mechanical pressure imposed on localized areas within the joints to a much larger tissue area, thus avoiding damage to localized (actually loaded) cartilage sites. Besides its mechanical importance, the extracellular matrix represents the microenvironment for the chondrocytes. The cells depend on the interaction with this pericellular matrix in order to keep their cellular phenotype stable. Also, the extracellular matrix is a major storage place for growth factors and possibly also for cytokines. These factors might be released and become functionally active upon enzymatic matrix degradation.

Unlike the first impression of a more or less static tissue, articular cartilage shows - albeit in the adult organism - a slow tissue turnover and remodelling (fig. 1). In foetal chondroneogenesis there is a significant net synthesis of cartilage matrix. There is a clear imbalance of matrix remodelling in favour of anabolism and, thus, growth of the cartilage tissue. In the adult cartilage, because of enzymatic activities and the mechanical stress imposed onto the joints, molecular damage always occurs. In normal circumstances this is compensated by the turnover of the matrix components, predominantly of the damaged proteoglycans. Thus, in normal adult articular cartilage there is an equal balance between anabolism and catabolism. If catabolism and molecular wear becomes stronger than the anabolic activities and capacities of the cartilage cells, the cartilage matrix degenerates and the joint cartilage gets damaged (fig. 1). This degeneration is known as osteoarthritis (OA). Depending on the pathogenesis, OA is divided into primary OA - which occurs without any known increase in damaging factors 


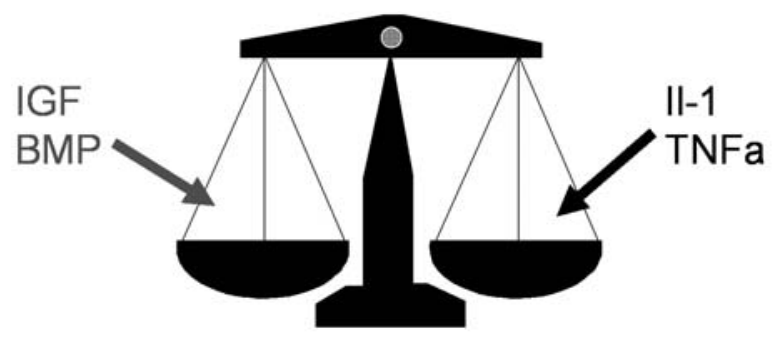

anabolism

aggrecan

collagen type $\mathrm{VI}$

collagen type IX

link protein catabolism MMP-2,9 ADAMTS-1 MMP-3 ADAMTS-4 MMP-13 ADMATS-5 MMP-14
Fig. 1: Osteoarthritis exhibits an imbalance in cartilage matrix turnover. The cells of the tissue (i.e. the chondrocytes) represent the active players within the process: they synthesize most of the matrix degrading proteases and fully provide anabolic activity. Even most of the anabolic and catabolic stimulatory factors, many of them not yet fully identified, are synthesized by the chondrocytes themselves in an auto- and paracrine manner.

(BMP: bone morphogenetic protein; IGF: insulin-like growth factor; Il-1ß: interleukin $1 \beta$; TNF $\alpha$ : tumour necrosis factor $\alpha$ )

- and secondary OA that is the result of an obvious condition, e. g. from multiple traumata, infections, synovial inflammation, and bone conditions (bone infarction, Paget's disease).

The initial stages of cartilage damage are characterized by a loosening of the collagen network and a loss of proteoglycans starting in the superficial and upper middle zones. This extends to deeper cartilage layers as the OA joint degeneration progresses. This type of damage is at least potentially reversible, also due to the possibility of an effective turnover of the proteoglycans in the adult tissue. After the loss of proteoglycans the now less elastic cartilage is more susceptible to molecular damage of the collagen network, which leads to the progressive deterioration of the articular cartilage integrity. Overall, this ongoing molecular destruction of the cartilage matrix is mainly driven by an increased activity of now partly identified proteases: the matrixmetalloproteinases (MMPs$2,-3,-13, \ldots)$ and the so called aggrecanases of the ADAMTS-family of proteases (most likely ADAMTS-4 and -5). These enzymes are induced by catabolic cytokines such as IL-1 or TNF $\alpha$, but also by matrix degradation products (Homandberg et al., 1998; Yasuda et al., 2002) and other non-physiologic stimuli, which the cells are exposed to. Recently, also epigenetic regulation of protease expression was reported in osteoarthritic versus normal articular cartilage (Roach et al., 2005).

Altogether, one of the most important questions in $\mathrm{OA}$ research is to understand the balance of catabolic and anabolic factors in articular cartilage, as this is the key to understanding the biology of cartilage maintenance and degeneration - and the basis of potential therapeutic interventions to prevent or even delay or stop the destruction process.

\section{Bone morphogenetic proteins up-regulate anabolic activity of chondrocytes}

As delineated above, anabolism is vital for cartilage to maintain its integrity - as the articular cartilage is subjected to mechanical stress and constant (or increased) catabolic proteolysis. Bone morphogenetic proteins (BMPs) are considered to be among the most potent anabolic factors for articular cartilage. They belong to the TGF- $\beta$ superfamily. Since their discovery by Urist and Wozney (Wozney et al., 1988; Yeomans et al., 1967) as factors, which can induce ectopic bone formation, multiple functions of BMPs in foetal development of the bone, the cartilage, the kidneys and the neural system have been identified (for review see Kishigami and Mishina, 2005). Experiments with knockout mice for various BMPs showed an embryonic lethal phenotype, or lead among other defects to severe malformations of the skeleton. In recent years experimental evidence accumulated that BMPs are not only important for the development of the articular cartilages, but are also present and active in the adult articular cartilage. A joint-specific GDF5-CreLoxP mouse model, which causes a knockout of the BMP receptor 1a and, therefore, blocks the effects of BMPs, showed severe OA after initially normal joint and cartilage development (Rountree et al., 2004). This suggests at least some importance of BMPs for the maintenance of adult cartilage beyond initial cartilage and joint formation in the embryonic period. Also, knock-down experiments using BMP-7 antisense oligonucleotides reduced aggrecan gene expression in adult cartilage and lead to proteoglycan depletion of the cartilage matrix (Soeder et al., 2005) (fig. 2). These data further support an important role of BMPs in cartilage homeostasis in the adult joint. In vitro, it has been well demonstrated that BMPs enhance proteoglycan and collagen type II synthesis (Fan et al., 2004b; Flechtenmacher et al., 1996; Goldring, 2000), a feature
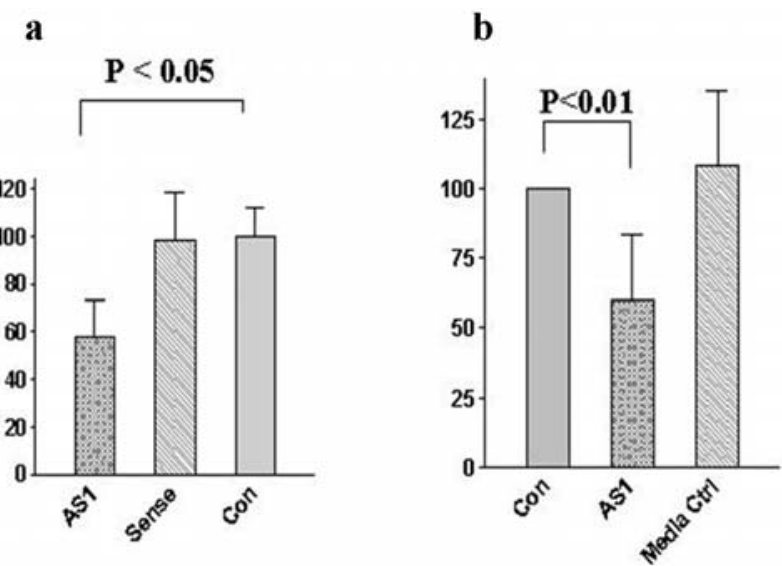

Fig. 2: Inhibition of BMP-7-dependent aggrecan mRNA expression (a) / proteoglycan synthesis (b) in adult human articular chondrocytes by BMP-7antisense treatment (AS1). Treatment with Sense showed no reduction in mRNA levels. Values in the control groups were defined as $100 \%$.

(Reproduced with kind permission from Soeder et al., 2005.) 
backed up by in vivo experiments (Glansbeek et al., 1997; Van Beuningen et al., 1998). Also, BMPs are thought to support the cellular phenotype of articular chondrocytes (Lackie, 1996; Sailor et al., 1996) and to counteract catabolic agents such as IL-1: thus, BMP-7 was able to counteract MMP-13 induction by IL-1ß in vitro (Im et al., 2003).

At present, it is unclear which BMPs are present and relevant in normal (and diseased) articular cartilage. So far, BMP-2 and BMP-7 are the best characterized members of the BMP protein family. Both show largely similar effects on chondrocyte anabolism as well as phenotype stabilization. In contrast to BMP-2, which appears to be increased in OA cartilage (Fukui et al., 2003), BMP-7 activity is, however, down-regulated in this condition (Merrihew et al., 2003a): whether this is one reason for the enhanced cartilage degeneration in OA needs further investigations.

Also unclear, at the moment, is whether OA chondrocytes are anabolically insufficiently activated (e.g. due to insufficient anabolic stimulation): in fact many data point to the opposite. However, even if chondrocytes in OA are generally anabolically hyperactive they are not able to compensate for the pronounced catabolic events occuring in OA cartilage, which ultimately destroy the articular cartilage. Local reductions in matrix synthesis are also found in the upper zones of osteoarthritic cartilage (Aigner et al., 1997). Thus, with their ability to increase matrix synthesis, BMPs could be at least potentially provide a therapeutic principle to be used to adjust the rate of synthetic activity of chondrocytes, in order to slow down cartilage destruction.

\section{Interleukin-1 down-regulates anabolic and up- regulates catabolic activity in articular chondrocytes}

Cartilage integrity is not only influenced by anabolic factors such as BMPs, but also by catabolic factors. One of the most prominent catabolic cytokines, supposed to play an important role also in $\mathrm{OA}$, is the pro-inflammatory cytokine interleukin 1 (IL-1) (Goldring, 1999; Goldring, 2000).

IL-1 is found at elevated levels in synovial fluids of patients suffering from rheumatoid arthritis and - to a lesser extent - of OA patients (Westacott et al., 1996). An increased presence of IL-1ß in OA cartilage has been reported using immunolocalization technology (Tetlow et al., 2001). Although we could not confirm an increased expression of IL-1 in OA chondrocytes by sensitive PCR (Fan et al., 2004a), the reported data could still reflect an increased presence of IL-1 protein in OA cartilage. IL-1 has multiple effects on articular chondrocytes: it downregulates the expression of the most abundant cartilage matrix components aggrecan and collagen type II (Goldring et al., 1988; Lefebvre et al., 1990; Richardson et al., 2000) and, thus, counteracts the effects of anabolic factors on matrix synthesis. Additionally, IL-1 is inducing the expression of matrix degrading enzymes such as MMP1 (collagenase 1), MMP-3 (stromelysin 1), MMP-13 (collagenase 3 ) or ADAMTS-4 (aggrecanase 1), which are potential major players in the destruction of cartilage matrix components (Bau et al., 2002; Mengshol et al., 2000; Richardson et al., 2000; Lefebvre et al., 1990). Besides its direct effects IL-1 also induces other cytokines with synergistic effects such as IL-6 and LIF (leukaemia inducing factor) (Fan et al., 2004a; Bender et al., 1990; Geng et al., 1996). IL-6 is in its effects similar to IL-1, but shows an overall weaker potential to inhibit proteoglycan synthesis (Guerne et al., 1999). It is needed for prolonged proteoglycan suppression by IL-1 (Nietfeld et al., 1990) and enhances MMP and aggrecanase expression and activity in articular cartilage (Flannery et al., 2000; Kusano et al., 1998). LIF, a protein belonging to a family of IL-6 related proteins, is also a suppressor of proteoglycan expression in articular chondrocytes and is able to increase MMP-13 production (Bell et al., 1995; Varghese et al., 1999).

Besides the knowledge of the effect of IL-1 on cellular gene expression and the understanding of the molecular mechanisms of IL-1, signal transduction in chondrocytes - including possible crosstalks - is increasingly important. In particular, this can expose targets specifically to modify adverse effects of IL-1ß without affecting the whole spectrum of physiological function of this molecule within intact cartilage, which might be functionally important as discussed below. These molecular mechanisms of IL-1 action have been studied extensively in other cell systems. IL-1 acts through the three classical MAPK-signalling pathways, namely ERK (extracellular signal regulated kinase), p38, and JNK (Jun terminal kinase), as well as NFkB (nuclear factor of kappa light polypeptide gene enhancer in B-cells) (for review see Saklatvala, 2006) (fig. 3). Of interest, whereas IL-1ß can activate all four pathways, the regulation of specific genes involves only a subset of these and this to a variable extent. Thus, the ERKpathway appears to be particularly important for the induction of other cytokines such as IL-6 and LIF (Fan et al., 2004a), whereas ERK and NFkB together regulate anabolic and catabolic genes such as collagen type II and MMP-1 and -13 (Barchowsky et al., 2000; Mengshol et al., 2000; Fan et al., 2006). The latter appears also to implicate signalling through $\mathrm{p} 38$.

\section{Is IL-1 important for the homeostasis of articular cartilage?}

At first glance, IL- 13 seems to have only deteriorating effects on the integrity of articular cartilage - due to the down-regulation of anabolic genes and induction of matrix degradation, but this picture possibly is not completely true. A study of knockout mice, in which IL-1ß function was abolished by inactivation of the IL- $1 \beta$ gene itself or indirectly by inactivation of the IL-1ß convertase (ICE) which is needed for IL- $1 \beta$ processing - showed that these knockout mice develop spontaneous $\mathrm{OA}$ at a higher rate than the wild types (Clements et al., 2003). Though these results are under debate (Glasson et al., 2005), an interesting question is: what could be the molecular mechanism for a potentially beneficial effect of IL-1 on 
articular cartilage? Recently, Sandell and colleagues were able to show that IL- $1 \beta$ and TNF $\alpha$ are potent inducers of BMP-2 expression, whereas the anabolic cytokines TGF 3 and IGF had no effect on the synthesis of BMP-2 (Fukui et al., 2003) (fig. 4). Similar effects, though to a lesser extent, were also described for IL-1 regarding BMP-7 (Merrihew et al., 2003b). Therefore, pro-inflammatory cytokines such as IL-1ß might induce anabolic effects, at least under some circumstances, physiologically as well as in cartilage pathology.

What could be the physiological role of this IL-1-BMP interaction in normal articular cartilage? It is intriguing to speculate that this interaction might be involved in the micromolecular repair of the extracellular matrix following molecular microdamage either due to (over)loading or enzymatic degradation. After inducing MMPs, which digest and remove damaged matrix components and pave the way for the deposition of new functional matrix components, IL-1 would - in this scenario - induce a compensatory anabolic activity of chondrocytes by an induction of anabolic growth factors, namely BMPs. Though this scenario still requires experimental confirmation, these results suggest that at least under some circumstances IL-1ß (and/or TNF $\alpha$ ) might be helpful for the cartilage to balance anabolic and catabolic effects in order to achieve matrix homeostasis. It is also tempting to speculate whether OA might be, at least in part, due to the over-activation or dysregulation of such processes which are, under normal conditions, helpful and possibly necessary for maintaining cartilage integrity.

\section{Intracellular crosstalk of BMPs and IL-1ß signalling pathways}

The effects of both anabolic and catabolic cytokines such as BMPs and IL- $1 \beta$ are mediated by different signalling pathways within the cells. In the case of BMPs the main components of the signalling pathway are the BMP receptors and the R-Smads (receptor-associated Smads 1, 5 and 8 ) as well as other members of the Smad family (for review see Massague et al., 2005) (fig. 5). Although, at first sight, the BMP (Smad) and IL-1 (ERK-p38-JNK/ $\mathrm{NFkB}$ ) pathways appear to be well separated, they are in fact cross-linked in several ways: Thus, BMPs/TGFßs can activate MAPkinases (namely p38) via Tak1 (TGF-ß activated kinase) (Kimura et al., 2000; Sano et al., 1999). Also, R-Smads can interact directly with target transcription factor of the MAPK-signalling system, such as the transcription factor c-Jun (Dennler et al., 2000), in order to modulate gene promoter activity. On the other hand, ERK-MAPkinases are able to phosphorylate RSmads in the linker region of these molecules. This prevents their nuclear translocation after receptor activation and thus, BMP signal transduction (Kretschmar et al., 1997; Pera et al., 2003). Another example shown for human articular chondrocytes, but known also from other cellular systems (Bitzer et al., 2000), is the induction of intracellular BMP-signalling inhibiting factors, namely the I(nhibitory)-Smad 7 by IL-1 (Kaiser et al., 2004).

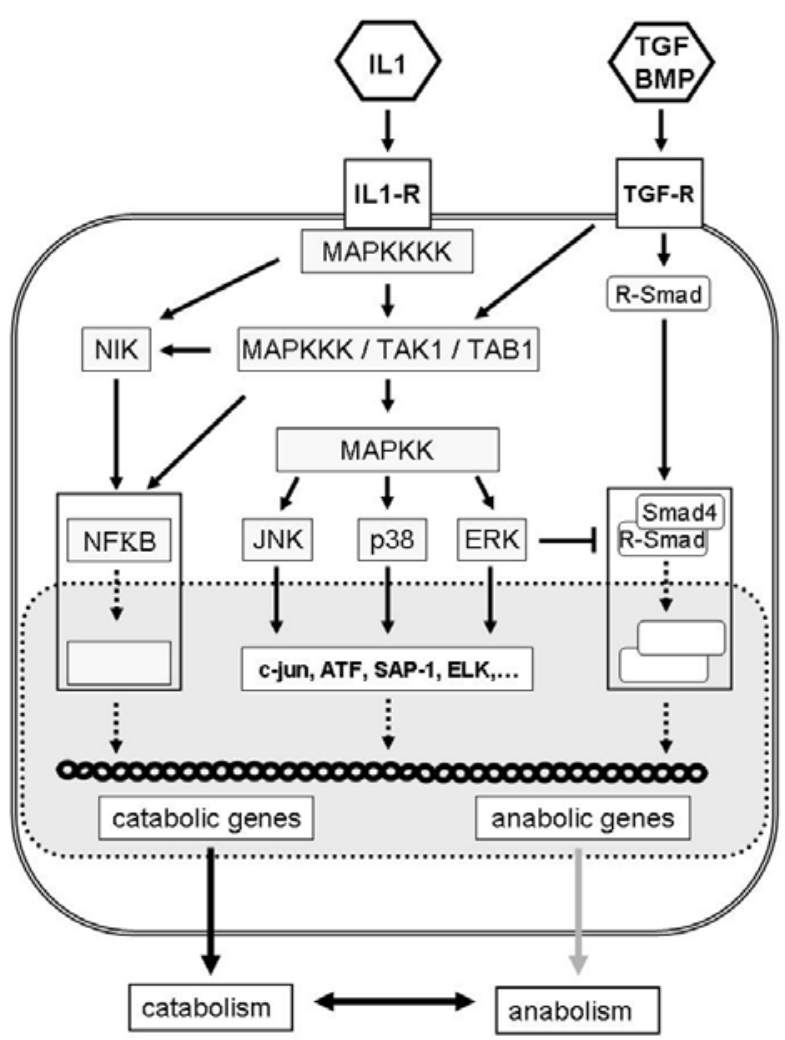

Fig. 3: Schematic representation of the IL-1 signalling pathway and some of its interactions with the BMP/ TGFß-signalling cascade.

Recently, performing a yeast-two-hybrid screen for RSmads 1 and 5, we found another potential candidate hinting at a cross-interaction of molecules involved in intracellular signalling of IL-1 and BMPs: Jab1 (Haag and Aigner, manuscript in preparation). Jab1 was originally described as a co-activator of c-jun/AP-1 activity (Tomoda et al., 1999), one major intracellular mediator of IL-1 activity. Jab1 interacts with c-jun and JunD (though not vjun and JunB) and enhances AP-1 activity by stabilizing c-jun and JunD binding to the AP-1 complex (Kleemann et al., 2000; Seeger et al., 1998). In our studies, we found Jab1 to interact physically with BMP R-SMAD 5. This binding resulted in an inhibition of BMP signalling. A similar mode of action was previously described for Jab1 regarding Smad 4, which further confirms the potential of Jab1 to modulate BMP-signalling.

Most likely, the so far characterized "cross-talks" in between anabolic and catabolic signalling pathways are only the "tip of the iceberg" and that there are numerous other, so far unknown, interaction levels. It remains an enormous task to define which of these potential interactions is active in vivo and especially, which interactions and interactors are relevant for $\mathrm{OA}$ development and progression. If it is possible to define these interactions they will provide potential key targets for prevention and manipulation of catabolic/anabolic gene regulation and therefore, the $\mathrm{OA}$ disease process. Especially, it would be possible to address special adverse effects of individual signalling cascades, allowing for a minimum of side effects. 

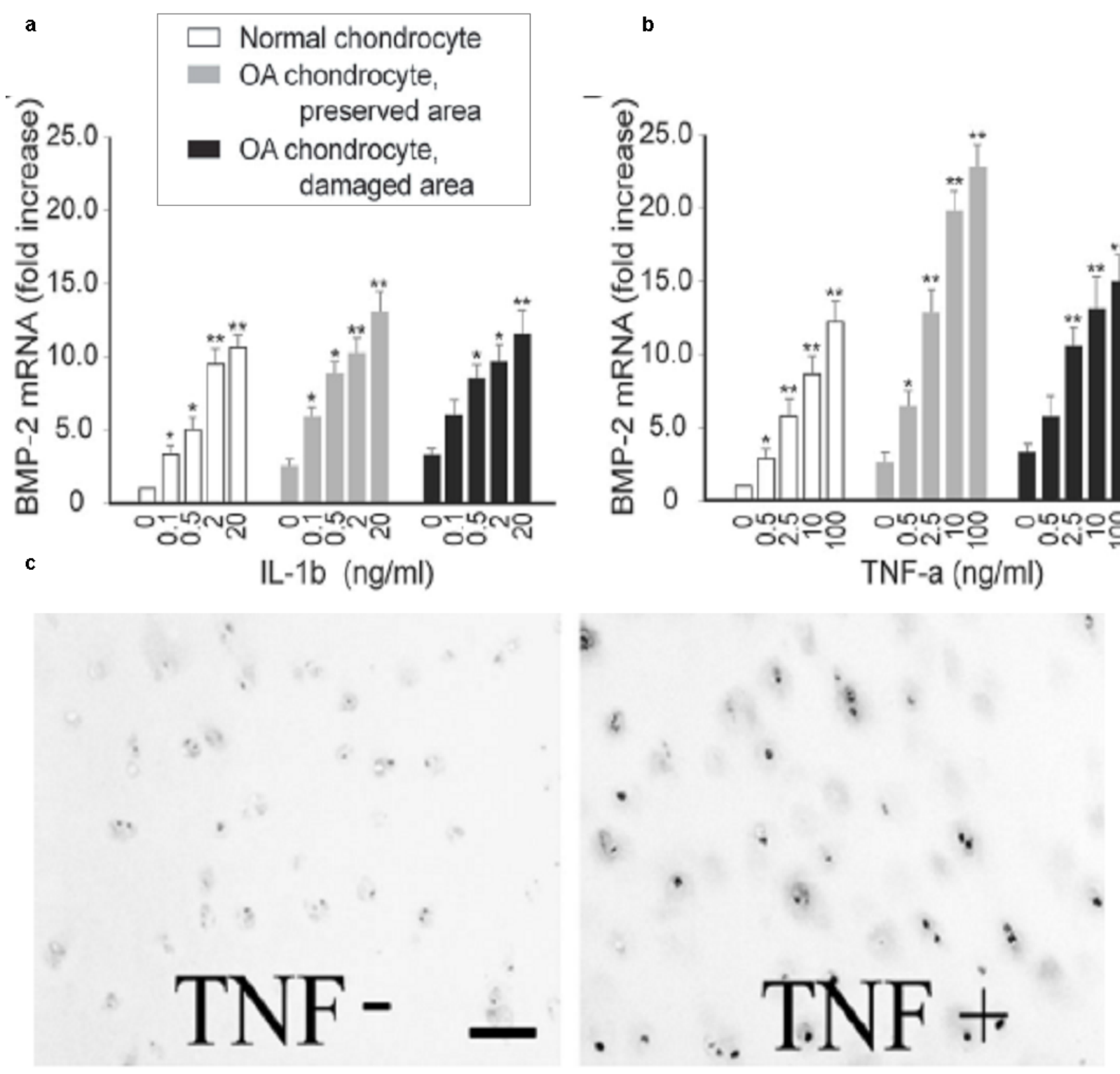

Fig. 4: BMP-2 is induced in normal and osteoarthritic (OA) human articular chondrocytes by IL-1ß and TNF $\alpha$ (a, b: cell culture; c: tissue culture).

(Reproduced with kind permission from Fukui et al. 2003)

\section{The anabolic - catabolic balance in articular cartilage - Final remarks}

Clearly, anabolic and catabolic pathways are very much intermingled in articular cartilage, as exemplified in this paper addressing IL-1 and BMP interaction. This anaboliccatabolic balance is titrated on numerous levels, starting from the mediator-synthesizing cells - which express either catabolic or anabolic factors - and ending up within the extracellular matrix itself, with constant molecular replacement and molecular destruction. Also, anabolic and catabolic gene expression compete with each other to provide a balance of matrix homeostasis: i.e. the more aggrecan is synthesized, the more aggrecanase activity is tolerable and even beneficial; the more aggrecanase is active, the more aggrecan needs to be synthesized by the articular chondrocytes in order to prevent matrix depletion and destruction. IL-1 and BMPs do enhance both (anabolic and catabolic) gene classes and are, consequently, influencing directly - by means of gene induction - this anabolic-catabolic balance in articular chondrocytes. Additionally, IL-1 more than BMPs, also down-regulates many effector genes of its counterpart (thus, IL-1 is downregulating anabolic genes such as aggrecan and collagen type II expression). In a next step, there are multiple layers of intracellular cross-talks in between the anabolic and catabolic signalling pathways, which are only in part yet established.

For cartilage homeostasis, a balance between anabolism (BMPs) and catabolism (IL-1) is necessary. This might allow the cartilage to adapt to challenges occurring during normal use of the joint. All this indicates how complex the dynamics of matrix and tissue homeostasis is. Maybe the most important lesson of this overview might be the notion that it is the balance, as such, which counts and not so much sufficient net anabolism or limited catabolism alone. 

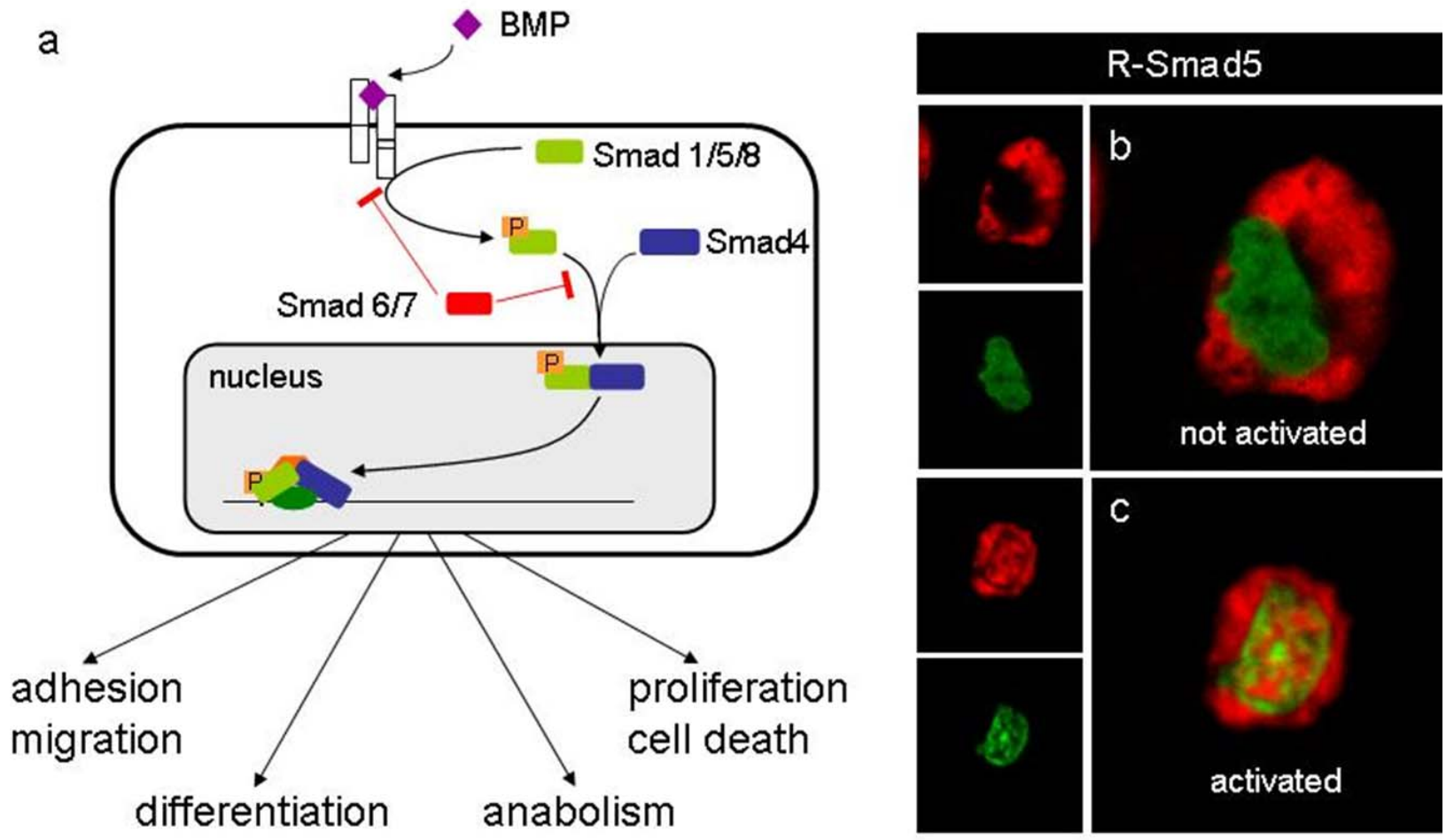

Fig. 5: The BMP signalling pathway (a). Induced by the binding of BMPs to their specific membrane receptors the BMP-specific receptor-associated R-Smad proteins (Smad 1, 5 and 8) become phosphorylated at specific sites at their C-terminus. This allows the R-Smads to form complexes with the common Smad protein, Smad4, and to translocate into the nucleus (see nuclear staining of Smad-5 (green) in c in contrast to b (both normal articular chondrocytes; red: nuclear DNA)), where the activated Smad proteins can form further complexes with transcriptional cofactors (coativators or corepressors). The exact composition of these complexes determines the nature of the cellular response, which can range from proliferation and differentiation to cell death. The BMP signalling pathway is negatively controlled by the inhibitory Smad proteins, Smad 6 and 7, which compete with the R-Smads for interaction with the activated receptors and/or the common Smad4, thereby preventing the formation of activated Smad complexes.

Central to the matter, might be the interwoven dynamics of the anabolic-catabolic balance, possibly suggested most intriguingly - by the effects of IL-1 on articular cartilage. A pure increase of anabolism and pure blockage of catabolism might not be the ultimate goal of OA therapy. Instead, the balanced anabolic-catabolic activities, as a whole, might require proper regulation and balancing.

\section{Acknowledgments}

This work was supported by the DFG (grant Ai20/7-1)

\section{References}

Aigner T, McKenna LA (2002) Molecular pathology and pathobiology of osteoarthritic cartilage. Cell Mol Life Sci 59: 5-18.

Aigner T, Vornehm SI, Zeiler G, Dudhia J, von der Mark K, Bayliss MT (1997) Suppression of cartilage matrix gene expression in upper zone chondrocytes of osteoarthritic cartilage. Arthritis Rheum 40: 562-569.

Barchowsky A, Frleta D, Vincenti MP (2000) Integration of the NF-kappaB and mitogen-activated protein kinase/AP-1 pathways at the collagenase-1 promoter: divergence of IL-1 and TNF-dependent signal transduction in rabbit primary synovial fibroblasts. Cytokine 12: 1469-1479.

Bau B, Gebhard PM, Haag J, Knorr T, Bartnik E, Aigner T (2002) Relative messenger RNA expression profiling of collagenases and aggrecanases in human articular chondrocytes in vivo and in vitro. Arthritis Rheum 46: $2648-2657$.

Bell MC, Carroll GJ (1995) Leukaemia inhibitory factor (LIF) suppresses proteoglycan synthesis in porcine and caprine cartilage explants. Cytokine 7: 137-141.

Bender S, Haubeck HD, van de Leur E, Dufhues G, Schiel X, Lauwerijns J, Greiling H, Heinrich PC (1990) Interleukin-1 beta induces synthesis and secretion of interleukin-6 in human chondrocytes. FEBS Lett 263: 321324.

Bitzer M, von Gersdorff G, Liang D, DominguezRosales A, Beg AA, Rojkind M, Bottinger EP (2000) A mechanism of suppression of TGF-beta/SMAD signaling by NF-kappa B/RelA. Genes Dev 14: 187-197.

Clements KM, Price JS, Chambers MG, Visco DM, Poole AR, Mason RM (2003) Gene deletion of either interleukin-1beta, interleukin-1beta-converting enzyme, inducible nitric oxide synthase, or stromelysin 1 accelerates 
the development of knee osteoarthritis in mice after surgical transection of the medial collateral ligament and partial medial meniscectomy. Arthritis Rheum 48: 3452-3463.

Dennler S, Prunier C, Ferrand N, Gauthier JM, Atfi A (2000) c-Jun inhibits transforming growth factor betamediated transcription by repressing $\mathrm{Smad} 3$ transcriptional activity. J Biol Chem 275: 28858-28865.

Fan Z, Bau B, Yang H, Aigner T (2004a) Il-beta induction of Il-6 and LIF in normal articular human chondrocytes involves the ERK, p38 and NFkB signaling pathways. Cytokine 28: 17-24.

Fan Z, Chubinskaya S, Rueger D, Bau B, Haag J, Aigner T (2004b) Regulation of anabolic and catabolic gene expression in normal and osteoarthritic adult human articular chondrocytes by osteogenic protein-1. Clin Exp Rheumatol 22: 103-106.

Fan Z, Yang H, Bau B, Soder S, Aigner T (2006) Role of mitogen-activated protein kinases and NFkappaB on IL-1 beta-induced effects on collagen type II, MMP-1 and 13 mRNA expression in normal articular human chondrocytes. Rheumatol Int In press.

Flannery CR, Little CB, Hughes CE, Curtis CL, Caterson B, Jones SA (2000) IL-6 and its soluble receptor augment aggrecanase-mediated proteoglycan catabolism in articular cartilage. Matrix Biol 19: 549-553.

Flechtenmacher J, Huch K, Thonar EJMA, Mollenhauer J, Davies SR, Schmid T, Puhl W, Sampath TK, Aydelotte MB, Kuettner KE (1996) Recombinant human osteogenic protein 1 is a potent stimulator of the synthesis of cartilage proteoglycans and collagens by human articular chondrocytes. Arthritis Rheum 39: 18961904.

Fukui N, Zhu Y, Maloney WJ, Clohisy J, Sandell LJ (2003) Stimulation of BMP-2 expression by proinflammatory cytokines IL-1 and TNF-alpha in normal and osteoarthritic chondrocytes. J Bone Joint Surg Am 85-A Suppl 3: 59-66.

Geng Y, Valbracht J, Lotz M (1996) Selective activation of the mitogen-activated protein kinase subgroups c-Jun NH2 terminal kinase and p38 by IL- 1 and TNF in human articular chondrocytes. J Clin Invest 98: 2425-2430.

Glansbeek HL, Van Beuningen HM, Vitters EL, Morris EA, van der Kraan P, van den Berg WB (1997) Bone morphogenetic protein stimulates articular cartilage proteoglycan synthesis in vivo but does not counteract interleukin-1a effects on proteoglycan synthesis and content. Arthritis Rheum 40: 1020-1028.

Glasson SS, Blanchet TJ, Morrris EA (2005) Less severe osteoarthritis is observed in Il-1b knock-out mice and more severe osteoarthritis is observed in MMP9 and MK2 knocked-out mice in a surgical model of osteoarthritis. 51st Annual Meeting of the ORS: 251.

Goldring MB (1999) The role of cytokines as inflammatory mediators in osteoarthritis: lessons from animal models. Connective Tissue Research 40: 1-11.

Goldring MB (2000) Osteoarthritis and Cartilage: The Role of Cytokines. Curr Rheumatol Rep 2: 459-465.

Goldring MB, Birkhead JR, Sandell LJ, Kimura T, Krane SM (1988) Interleukin 1 suppresses expression of cartilage-specific types II and IX collagens and increases types I and III collagens in human chondrocytes. J Clin Invest 82: 2026-2037.

Guerne P-A, Desgeorges A, Jaspar J-M, Relic B, Peter R, Hoffmayer P, Dayer J-M (1999) Effects of IL-6 and its soluble receptor on proteoglycan synthesis and NO release by human articular chondrocytes: comparison with IL-1. Modulation by dexamethasone. Matrix Biol 18: 253-260.

Homandberg GA, Wen C, Hui F (1998) Cartilage damaging activities of fibronectin fragments derived from cartilage and synovial fluid. Osteoarthritis Cartilage 6: 231244.

Im HJ, Pacione C, Chubinskaya S, Van Wijnen AJ, Sun Y, Loeser RF (2003) Inhibitory effects of insulin-like growth factor-1 and osteogenic protein-1 on fibronectin fragment- and interleukin-1beta-stimulated matrix metalloproteinase-13 expression in human chondrocytes. J Biol Chem 278: 25386-25394.

Kaiser M, Haag J, Söder S, Bau B, Aigner T (2004) BMP- and TGFß-inhibitory Smads 6 and 7 are expressed in human adult normal and osteoarthritic cartilage in vivo and differentially regulated in vitro by Il-1ß. Arthritis Rheum 11: 3535-3540.

Kimura N, Matsuo R, Shibuya H, Nakashima K, Taga T (2000) BMP2-induced apoptosis is mediated by activation of the TAK1-p38 kinase pathway that is negatively regulated by Smad6. J Biol Chem 275: $17647-$ 17652.

Kishigami S, Mishina Y (2005) BMP signaling and early embryonic patterning. Cytokine Growth Factor Rev 16: $265-278$.

Kleemann R, Hausser A, Geiger G, Mischke R, BurgerKentischer A, Flieger O, Johannes FJ, Roger T, Calandra T, Kapurniotu A, Grell M, Finkelmeier D, Brunner H, Bernhagen J (2000) Intracellular action of the cytokine MIF to modulate AP-1 activity and the cell cycle through Jab1. Nature 408: 211-216.

Kretschmar M, Doody J, Massagué J (1997) Opposing bmp and egf signalling pathways converge on the tgf- $\beta$ family mediator smad 1. Nature 389: 618-622.

Kusano K, Miyaura C, Inada M, Tamura T, Ito A, Nagase H, Kamoi K, Suda T (1998) Regulation of matrix metalloproteinases (MMP-2, -3, -9, and -13) by interleukin-1 and interleukin-6 in mouse calvaria: association of MMP induction with bone resorption. Endocrinology 139: 1338-1345.

Lackie PM (1996) Immunogold silver staining for light microscopy. Histochem Cell Biol 106: 9-17.

Lefebvre V, Peeters-Joris C, Vaes G (1990) Modulation by interleukin 1 and tumor necrosis factor a of production of collagenase, tissue inhibitor of metalloproteinases and collagen types in differentiated and dedifferentiated articular chondrocytes. Biochim Biophys Acta 1052: 366378 .

Massague J, Seoane J, Wotton D (2005) Smad transcription factors. Genes Dev 19: 2783-2810.

Mengshol JA, Vincenti MP, Coon CI, Barchowsky A, Brinckerhoff CE (2000) Interleukin-1 induction of collagenase 3 (matrix metalloproteinase 13) gene expression in chondrocytes requires $\mathrm{p} 38$, c-Jun N-terminal kinase, and nuclear factor kappaB: differential regulation 
of collagenase 1 and collagenase 3 . Arthritis Rheum 43: 801-811.

Merrihew C, Kumar B, Heretis K, Rueger DC, Kuettner KE, Chubinskaya S (2003a) Alterations in endogenous osteogenic protein-1 with degeneration of human articular cartilage. J Orthop Res 21: 899-907.

Merrihew C, Soeder S, Rueger DC, Kuettner KE, Chubinskaya S (2003b) Modulation of endogenous osteogenic protein-1 (OP-1) by interleukin-1 in adult human articular cartilage. J Bone Joint Surg Am 85-A Suppl 3: 67-74.

Nietfeld JJ, Wilbrink B, Helle M, van Roy JLAM, Den Otter W, Swaak AJG, Huber-Bruning O (1990) Interleukin1 -induced interleukin- 6 is required for the inhibition of proteoglycan synthesis by interleukin-1 in human articular cartilage. Arthritis Rheum 33: 1695-1701.

Pera EM, Ikeda A, Eivers E, De Robertis EM (2003) Integration of IGF, FGF, and anti-BMP signals via Smad1 phosphorylation in neural induction. Genes Dev 17: 30233028.

Richardson DW, Dodge GR (2000) Effects of interleukin-1beta and tumor necrosis factor-alpha on expression of matrix-related genes by cultured equine articular chondrocytes. Am J Vet Res 61: 624-630.

Roach HI, Yamada N, Cheung KSC, Tilley S, Clarke NMP (2005) Abnormal expression of matrix-degrading enzymes by human osteoarthritic chondrocytes is associated with demethylation of specific CpGs in the promotor. Arthritis Rheum 52: 3110-3124.

Rountree RB, Schoor M, Chen H, Marks ME, Harley V, Mishina Y, Kingsley DM (2004) BMP Receptor Signaling Is Required for Postnatal Maintenance of Articular Cartilage. PLoS Biol 2: e355.

Sailor LZ, Hewick RM, Morris EA (1996) Recombinant human bone morphogenetic protein-2 maintains the articular chondrocyte phenotype in long-tem culture. J Orthop Res 14: 937-945.

Saklatvala J (2006) Inflammatory signalling in cartilage: MAPK and NF-B pathways in chondrocytes and the use of inhibitors for research into pathogenesis and therapy of osteoarthritis. Current Drug Targets In press.

Sano Y, Harada Y, Tashiro S, Gotoh-Mandeville R, Maekawa T, Ishii S (1999) atf-2 is acommon nuclear target of smad and tak1 pathways in transforming growth factorß signaling. J Biol Chem 274: 8949-8957.

Seeger M, Kraft R, Ferrell K, Bech-Otschir D, Dumdey R, Schade R, Gordon C, Naumann M, Dubiel W (1998) A novel protein complex involved in signal transduction possessing similarities to $26 \mathrm{~S}$ proteasome subunits. FASEB J 12: 469-478.

Soeder S, Hakimiyan A, Rueger D, Kuettner K, Aigner T, Chubinskaya S (2005) Antisense inhibition of osteogenic protein-1 disturbs human articular cartilage integrity. Arthritis Rheum 52: 468-478.

Tetlow LC, Adlam DJ, Woolley DE (2001) Matrix metalloproteinase and proinflammatory cytokine production by chondrocytes of human osteoarthritic cartilage: associations with degenerative changes. Arthritis Rheum 44: 585-594.

Tomoda K, Kubota Y, Kato J (1999) Degradation of the cyclin-dependent-kinase inhibitor p27Kip1 is instigated by Jab1. Nature 398: 160-165.

Van Beuningen HM, Glansbeek HL, van der Kraan P, van den Berg WB (1998) Differential effects of local application of BMP-2 or TGF- $\beta$ on both articular cartilage composition and osteophyte formation. Osteoarthritis Cartilage 6: 306-317.

Varghese S, Yu K, Canalis E (1999) Leukemia inhibitory factor and oncostatin M stimulate collagenase3 expression in osteoblasts. Am J Physiol 276: E465-E471.

Westacott CI, Sharif M (1996) Cytokines in osteoarthritis: mediators or markers of joint destruction? Semin Arthritis Rheum 25: 254-272.

Wozney JM, Rosen V, Celeste AJ, Mitsock LM, Whitters MJ, Kriz RW, Hewick RM, Wang EA (1988) Novel regulators of bone formation: Molecular clones and activities. Science 242: 1528-1534.

Yasuda T, Poole AR (2002) A fibronectin fragment induces type II collagen degradation by collagenase through an interleukin-1-mediated pathway. Arthritis Rheum 46: 138-148.

Yeomans JD, Urist MR (1967) Bone induction by decalcified dentine implanted into oral, osseous and muscle tissues. Arch Oral Biol 12: 999-1008.

\section{Discussion with reviewers}

M. Stoddart: It is known that human knee and ankle articular cartilage behave differently in response to IL-1 $\beta$. Have you looked into these differences, or do you know are any reason why these two similar tissues respond differently?

Authors: This point is absolutely true and not only inbetween different joints, but also in-between different individuals and ages. We, as all others, try mostly to follow up the main lines of evidence. Still there is excellent work presented by the Kuettner group (Chicago) showing clear differences in between the knee and the ankle joints regarding the responsiveness of cells to IL-1: the exact molecular mechanisms are still not fully understood though first progresses have been made (for review see Kerin et al., 2002). We ourselves have never performed comparative analyses.

\section{Additional Reference}

Kerin A, Patwari P, Kuettner K, Cole A, Grodzinsky A (2002). Related Articles, Molecular basis of osteoarthritis: biomechanical aspects. Cell Mol Life Sci 59: 27-35. 